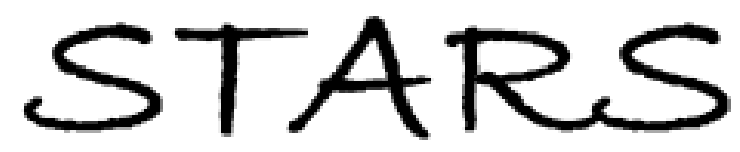

University of Central Florida

STARS

$1-1-2007$

\title{
Transflective liquid crystal display using commonly biased reflectors
}

\author{
Zhibing Ge \\ University of Central Florida \\ Xinyu Zhu \\ University of Central Florida \\ Robert Lu \\ University of Central Florida \\ Thomas X. Wu \\ University of Central Florida \\ Shin-Tson Wu \\ University of Central Florida
}

Find similar works at: https://stars.library.ucf.edu/facultybib2000

University of Central Florida Libraries http://library.ucf.edu

This Article is brought to you for free and open access by the Faculty Bibliography at STARS. It has been accepted for inclusion in Faculty Bibliography 2000 s by an authorized administrator of STARS. For more information, please contactSTARS@ucf.edu.

\section{Recommended Citation}

Ge, Zhibing; Zhu, Xinyu; Lu, Robert; Wu, Thomas X.; and Wu, Shin-Tson, "Transflective liquid crystal display using commonly biased reflectors" (2007). Faculty Bibliography 2000s. 7147.

https://stars.library.ucf.edu/facultybib2000/7147

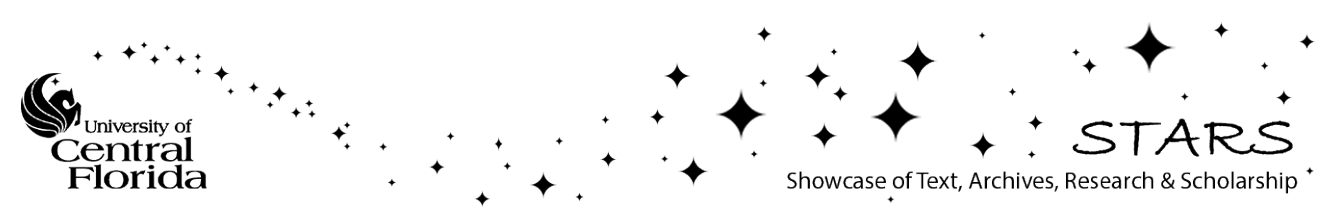




\section{Transflective liquid crystal display using commonly biased reflectors}

Cite as: Appl. Phys. Lett. 90, 22111 (2007); https://doi.org/10.1063/1.2745206

Submitted: 16 April 2007. Accepted: 07 May 2007. Published Online: 31 May 2007

Zhibing Ge, Xinyu Zhu, Robert Lu, Thomas X. Wu, and Shin-Tson Wu

ARTICLES YOU MAY BE INTERESTED IN

Wide-view and single cell gap transflective liquid crystal display using slit-induced multidomain structures

Applied Physics Letters 92, 191102 (2008); https://doi.org/10.1063/1.2926669

Low voltage blue-phase liquid crystal displays

Applied Physics Letters 95, 231101 (2009); https://doi.org/10.1063/1.3271771

Single cell gap and wide-view transflective liquid crystal display using fringe field switching and embedded wire grid polarizer

Applied Physics Letters 92, 051109 (2008); https://doi.org/10.1063/1.2841847

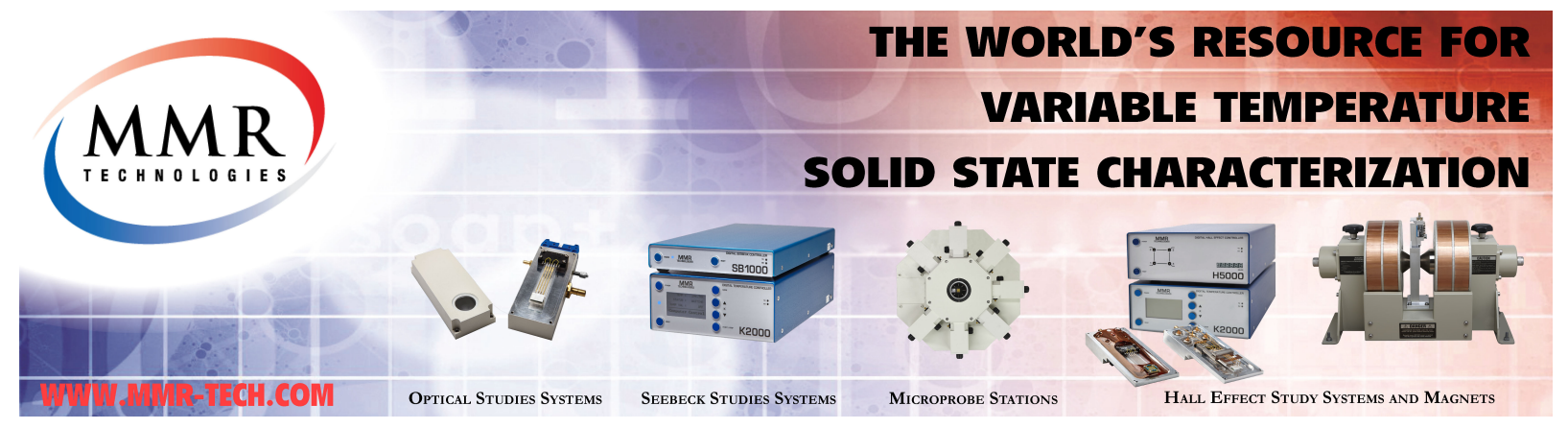




\title{
Transflective liquid crystal display using commonly biased reflectors
}

\author{
Zhibing Ge, Xinyu Zhu, Robert Lu, Thomas X. Wu, and Shin-Tson Wu ${ }^{\mathrm{a}}$ \\ College of Optics and Photonics, University of Central Florida, Orlando, Florida 32816
}

(Received 16 April 2007; accepted 7 May 2007; published online 31 May 2007)

\begin{abstract}
A normally black single cell gap transflective liquid crystal display (LCD) using vertical alignment and commonly biased patterned reflectors is developed. In the transmissive region, continuous and uniform vertical electric fields are formed throughout the LC cell. On the other hand, in the reflective region, the electric fields are mainly confined near the substrate surfaces. As a result, the phase retardation in the transmissive region is approximately two times as that of the reflective region. Such a transflective LCD shows a high light efficiency $(>90 \%)$ for both transmissive and reflective modes and well matched gray scales. (C) 2007 American Institute of Physics.
\end{abstract}

[DOI: $10.1063 / 1.2745206]$

Transflective liquid crystal displays (TR-LCDs) have been used extensively in mobile displays such as cell phones and personal digital assistants because of their low power consumption and good outdoor readability. ${ }^{1}$ In general, TR-LCDs can be divided into two categories based on the LC cell configurations: dual cell gap ${ }^{2,3}$ and single cell gap structures. ${ }^{4-9}$ The dual cell gap TR-LCDs employ different cell gaps in the transmissive $(T)$ region and the reflective $(R)$ region to compensate their optical path difference. As a result, high transmittance and high reflectance are obtained and their voltage-dependent transmittance $(V-T)$ and reflectance $(V-R)$ curves overlap quite well. However, the fabrication process is rather complicated and the response time for the $T$ and $R$ modes is different. On the other hand, the single cell gap TR-LCDs use the same cell gap for both $T$ and $R$ regions. Its fabrication is easier and the response time for the $T$ and $R$ modes is the same. Thus, this approach has attracted much research attention. The main technical challenges in single cell gap TR-LCDs are twofold: (1) it is difficult to obtain high $T$ and $R$ simultaneously and (2) the gray scale matching between the $T$ and $R$ modes is usually poor.

In this letter, we report a normally black single cell gap TR-LCD using a vertical alignment (VA) LC cell with special rubbing directions. This device uses commonly biased reflectors to generate different electric field distributions in the $T$ and $R$ regions. As a result, the device shows a high light efficiency and excellent match between $V-T$ and $V-R$ curves.

Figure 1 shows the schematic structure of the proposed transflective LCD device with commonly biased reflectors. On each side of the LC cell, a broadband quarter-wave plate $^{10}$ along with a linear polarizer works together as a circular polarizer. Within each pixel, the LC cell is repetitively divided into $T$ and $R$ regions. In the $T$ region, the LC layer is sandwiched between the top transparent indium tin oxide (ITO) common electrode and the bottom ITO pixel electrode. In the $R$ region, periodically patterned conductive layers are formed above the bottom pixel electrode as the reflectors, which are commonly biased to a certain voltage (below the threshold voltage) for all pixels. When no voltage is applied, all the LC directors are vertically aligned. Therefore, the light passing through the LC layer experiences no phase re-

\footnotetext{
${ }^{a)}$ Electronic mail: swu@ mail.ucf.edu
}

tardation and keeps its polarization state, resulting in a dark state for both $T$ and $R$ modes. On the other hand, as the voltage exceeds a threshold $\left(V_{\mathrm{th}}\right)$, the LC directors are tilted and the phase retardation effect takes place. Ideally, the required phase retardation change is $\sim \lambda / 2$ for the $T$ region and $\sim \lambda / 4$ for the $R$ region in order to achieve maximum transmittance and reflectance.

Figure 2 shows the calculated equal-potential line distribution in the LC layer within one repetitive period when $V=6.5 \mathrm{~V}_{\mathrm{rms}}$ is applied to the bottom pixel electrode, while all the reflectors are biased at $0 \mathrm{~V}_{\mathrm{rms}}$ The potential difference between neighboring vertical lines is $0.25 \mathrm{~V}_{\mathrm{rms}}$. Here the LC material employed is a negative dielectric anisotropy $(\Delta \varepsilon)$ (Merck MLC-6608), with its extraordinary and ordinary refractive indices $n_{e}=1.5578$ and $n_{o}=1.4748$ (at $\lambda=589 \mathrm{~nm}$ ), its parallel and perpendicular dielectric constants $\varepsilon_{\|}=3.6$ and $\varepsilon_{\perp}=7.8$, and its elastic constants $K_{11}=16.7 \mathrm{pN}$ and $K_{33}=18.1 \mathrm{pN}$. The cell gap of the LC layer is $5 \mu \mathrm{m}$, the reflector width is $2 \mu \mathrm{m}$, and the horizontal gap between adjacent reflectors is $5 \mu \mathrm{m}$. In order to show the relative positions, both vertical and horizontal dimensions in Fig. 2 are normalized to their real lengths. In the $T$ regions $(x \in[0,0.18],[0.32,0.68]$, and $[0.82,1])$ in Fig. 2 , the potential values decrease quite uniformly in the vertical directions, indicating a uniform distribution of vertical electric fields in those regions. However, in the $R$ regions $(x \in[0.18,0.32]$ and $[0.68,0.82])$, the regions with uniform potential changes in the vertical direction are located near the surfaces, e.g., from $z=0$ to $\sim 0.3$ and from $z=\sim 0.7$ to 1.0 . Correspondingly, strong vertical fields are only confined to the cell surface areas, and an area with ultralow vertical field components in the bulk LC layer is generated. This type of field distribution originates mainly from the voltage shielding by the commonly biased reflectors. Here we call this phenomenon as surface field effect. Under this field distribution, the LC directors in the $T$ region can tilt more than those in the $R$ region. That means that the effective phase retardation in the $T$ region is more than that in the $R$ region. This is what a transflective LCD needs.

Due to the existence of strong horizontal electric fields, as shown in Fig. 2, the initial surface rubbing directions of the LC directors play a critical role in affecting the transmittance. Figure 3 shows the calculated time-dependent transmittance using extended Jones matrix method ${ }^{11,12}$ for the 


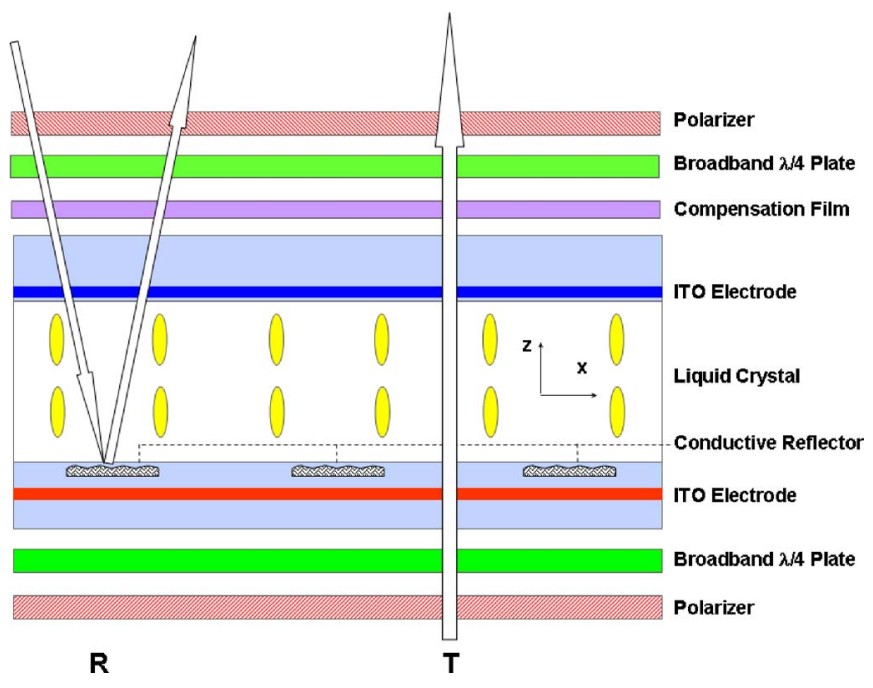

FIG. 1. (Color online) Schematic cell structure of the single cell gap transflective LCD using commonly biased conductive reflectors.

cases with surface rubbing angles $\phi=0^{\circ}$ (parallel), $\phi=50^{\circ}$, and $\phi=90^{\circ}$ (perpendicular) with respect to the strip electrodes in the $x$ direction. In these cases, the pretilt angle is $88^{\circ}$, the pixel electrode voltage is $6.5 \mathrm{~V}_{\mathrm{rms}}$, and the reflectors are all biased at $0 \mathrm{~V}_{\mathrm{rms}}$. From Fig. 3, when $\phi=50^{\circ}$ and $90^{\circ}$, the transmittance increases monotonously from $0 \%$ to over 90\% (normalized to the maximum light transmittance through two polarizers) and saturates when $t>80 \mathrm{~ms}$. This indicates that the LC directors experience a smooth reorientation and finally reach at a stable state. On the other hand, when $\phi=0^{\circ}$ (along the $+x$ direction in Fig. 1) the maximum transmittance is lower than those of $\phi=50^{\circ}$ and $90^{\circ}$ below $100 \mathrm{~ms}$, then two transition kinks occur at $t \sim 90 \mathrm{~ms}$ and $t \sim 120 \mathrm{~ms}$ and the transmittance further increases to a final saturation level. From Fig. 3, the LC reorientation mechanisms for these two cases are different.

To understand the rubbing angle effect, we need to know the LC director distribution for both cases. Figure 4(a) shows the calculated LC director distribution using 2DIMMOS software (from autronic-MELCHERS, Germany) at $t \sim 110 \mathrm{~ms}$ for the case of $\phi=0^{\circ}$. When a high voltage is applied, the

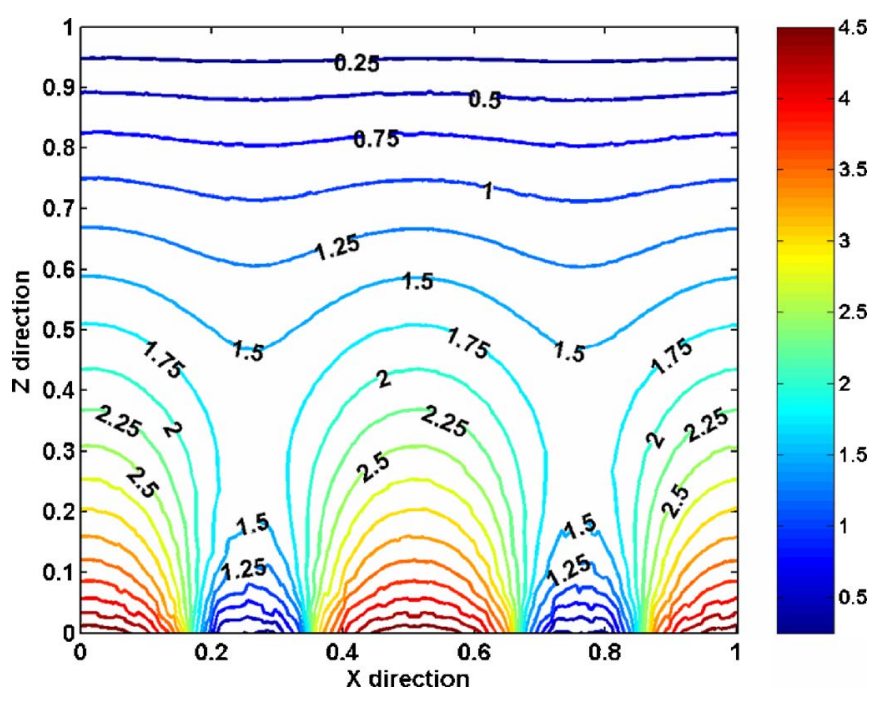

FIG. 2. (Color online) Equal-potential lines in the LC cell region for one sample section with $6 \mathrm{~V}_{\mathrm{rms}}$ applied to pixel electrode and the reflectors are commonly biased at $0 \mathrm{~V}_{\text {rms. }}$.

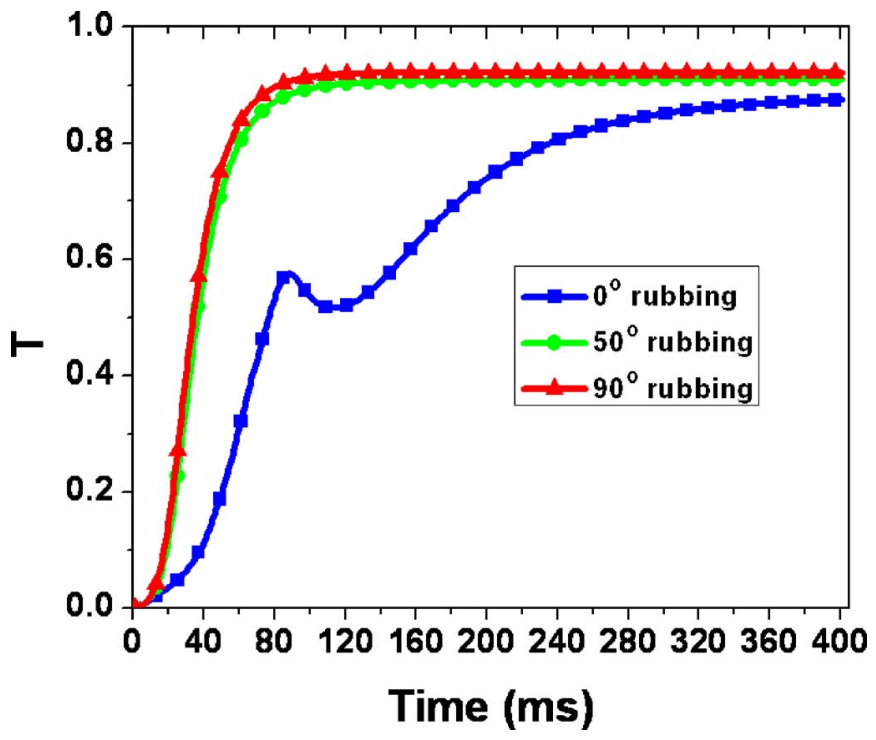

FIG. 3. (Color online) Time-dependent transmittance plots for cells at rubbing angles $\phi=0^{\circ}, 50^{\circ}$, and $90^{\circ}$.

vertical electric fields between the pixel and common electrodes and the horizontal fields at the right edge of the reflectors [position A in Fig. 4(a)] tend to tilt down the LC directors toward the $+x$ direction due to the $2^{\circ}$ pretilt angle. However, the horizontal electric fields at the left edge of the reflectors [position B in Fig. 4(a)] tend to reorient the LC directors toward the $-x$ direction. These two opposite torques

(a)
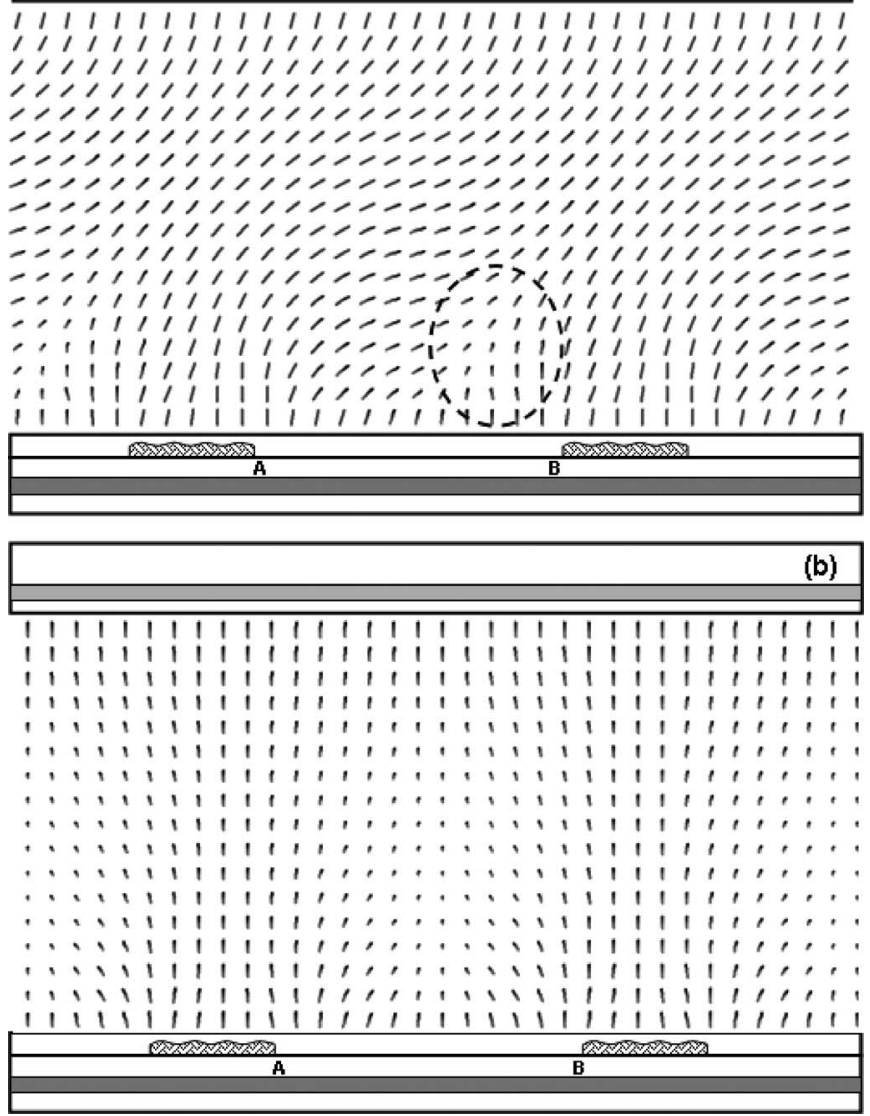

FIG. 4. LC director distribution with rubbing angles (a) $\phi=0^{\circ}$ at $t=110 \mathrm{~ms}$ and (b) $\phi=90^{\circ}$ at $t=110 \mathrm{~ms}$. 
push the $-\Delta \varepsilon$ LC molecules in their adjoining region [the circled region in Fig. 4(a)] to twist into the $+y$ direction. Thus a twisted region is formed and the light efficiency is lowered, as observed in Fig. 3, at $t \sim 100 \mathrm{~ms}$. This lower transmittance is because the twisted LC structure exhibits a lower transmittance when sandwiched between two circular polarizers. ${ }^{3}$ After $100 \mathrm{~ms}$, all the LC directors start to twist into the $+y$ direction and the transmittance increases again. However, this transition process takes a long time, as shown in Fig. 3.

If the initial rubbing direction is far away from the $x$ direction, such as $90^{\circ}$, a smoother LC reorientation occurs. Figure 4(b) shows the director distribution at $t=110 \mathrm{~ms}$ with $\phi=90^{\circ}$. Once a high voltage is applied, the vertical electric fields will substantially tilt the directors down into the $+y$ direction. Similarly, the horizontal field components at edges of the reflectors [positions A and B in Fig. 4(b)] tend to tilt the nearby LC directors toward the opposite directions. However, because most of LC directors have been tilted down into the $+y$ direction, their competence can be balanced. Consequently, the change of transmittance with respect to time is smooth and it reaches saturation much faster than that with an initial rubbing direction set at $0^{\circ}$. Similarly, for the cell with rubbing angle $\phi=50^{\circ}$, the preorientation by the surface rubbing also helps the LC directors tilt down smoothly and quickly into the rubbing direction by the vertical fields, as indicated in Fig. 3.

In the $R$ regions the electric fields are mainly confined to the surface regions, the LC directors near the top and bottom surfaces are first tilted down to the $+y$ direction when $\phi=90^{\circ}$. Because the LC is a continuum, their reorientation will further perturb the molecules in the low field region. Because of the existence of this low field region, the overall tilt of LC directors will be much lower than those in the $T$ region. By optimizing the dimensions of reflector width, gap, rubbing angle, and the commonly biased voltages on the reflectors, it is possible to obtain a high optical efficiency for the $T$ and $R$ modes and a small disparity between the $V-T$ and $V$ - $R$ curves.

Figure 5(a) shows the calculated $V-T$ and $V-R$ curves for a single cell gap transflective LCD with $5 \mu \mathrm{m}$ cell gap, $2 \mu \mathrm{m}$ reflector width, and $5 \mu \mathrm{m}$ reflector gap. The commonly biased voltage for the reflectors is $V=0 \mathrm{~V}_{\mathrm{rms}}$ for all pixels. The surface rubbing angle is at $90^{\circ}$ and the pretilt angle is $88^{\circ}$. Both $V-T$ and $V$ - $R$ curves reach $\sim 90 \%$ ( $T: \sim 91 \%$ and $R: \sim 93 \%$ ) light efficiency at $V=6.5 \mathrm{~V}_{\mathrm{rms}}$. Besides, the $V-T$ and $V$ - $R$ curves also show a reasonably good overlap with each other. To further reduce the difference between $V-T$ and $V-R$ curves, a small voltage $1.5 \mathrm{~V}_{\text {rms }}$ (it is below threshold and will not affect the dark state) is applied to the commonly biased reflectors; the reflector width and gap are set at 3 and $6 \mu \mathrm{m}$, respectively, and the rubbing angle is set at $50^{\circ}$ with respect to the $x$ axis. Figure 5 (b) shows the corresponding $V-T$ and $V$ - $R$ curves. Indeed, a better gray scale overlap is obtained. In addition, this design is quite tolerant to the possible cell gap change due to the formation of embossed reflectors in the cell.

In conclusion, we have demonstrated a single cell gap transflective LCD using a VA cell with commonly biased reflectors. The LC directors in the $T$ region are mainly reoriented by the fairly uniform longitudinal electric fields while those in the $R$ region are mainly driven by the surface fields
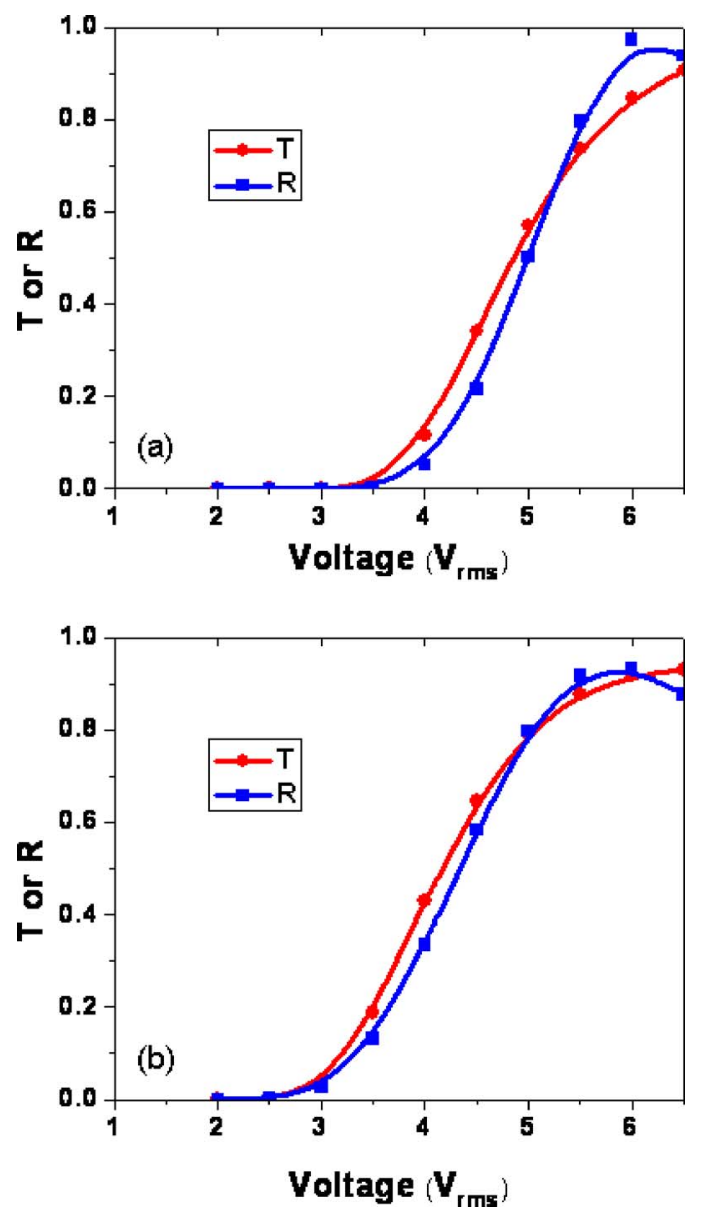

FIG. 5. (Color online) Voltage-dependent reflectance and transmittance curves for (a) $W=2 \mu \mathrm{m}, G=5 \mu \mathrm{m}, V_{\text {bias }}=0 \mathrm{~V}_{\text {rms }}$, and rubbing angle $\phi=90^{\circ}$ and (b) $W=3 \mu \mathrm{m}, G=6 \mu \mathrm{m}, V_{\text {bias }}=1.5 \mathrm{~V}_{\mathrm{rms}}$, and rubbing angle $\phi=50^{\circ}$.

which generate a less tilt in LC directors. As a result, the phase retardation in the $R$ region is nearly one-half as that in the $T$ region. Potential application of this transflective LCD for mobile displays, in which low power consumption and good outdoor readability are required, is foreseeable.

${ }^{1}$ S. T. Wu and D. K. Yang, Reflective Liquid Crystal Displays (Wiley, New York, 2001).

${ }^{2}$ M. Okamoto, H. Hiraki, and S. Mitsui, U.S. Patent No. 6,281,952 (28 August 2001).

${ }^{3}$ S. J. Roosendaal, B. M. I. van der Zande, A. C. Nieuwkerk, C. A. Renders, J. T. M. Osenga, C. Doornkamp, E. Peeters, J. Bruinink, J. A. M. M. van Haaren, and S. Takahashi, Soc. Inform. Display Symposium Digest 34, 78 (2003).

${ }^{4}$ X. Zhu, Z. Ge, T. X. Wu, and S. T. Wu, J. Disp. Technol. 1, 15 (2005).

${ }^{5}$ Y. J. Lee, H. R. Kim, T. H. Lee, J. W. Jung, J. H. Kim, and J. S. Choi, Soc. Inform. Display Symposium Digest 37, 821 (2006).

${ }^{6}$ Y. C. Yang, J. Y. Choi, J. Kim, M. Han, J. Chang, J. Bae, D. J. Park, S. I. Kim, N. S. Roh, Y. J. Kim, M. Hong, and K. Chung, Soc. Inform. Display Symposium Digest 37, 829 (2006).

${ }^{7}$ S. H. Lee, H. W. Do, G. D. Lee, T. H. Yoon, and J. C. Kim, Jpn. J. Appl. Phys., Part 2 42, L1455 (2004).

${ }^{8}$ Y. J. Lim, J. H. Song, Y. B. Kim, and S. H. Lee, Jpn. J. Appl. Phys., Part 2 43, L972 (2004).

${ }^{9}$ J. H. Song, Y. J. Lim, M. H. Lee, S. H. Lee, and S. T. Shin, Appl. Phys. Lett. 87, 011108 (2005).

${ }^{10}$ S. Pancharatnam, Proc. Indian Acad. Sci., Sect. A 41, 130 (1955).

${ }^{11}$ A. Lien, Appl. Phys. Lett. 57, 2767 (1990).

${ }^{12}$ Z. Ge, X. Zhu, T. X. Wu, and S. T. Wu, J. Opt. Soc. Am. A 22, 966 (2005). 